\title{
Using food models to enhance sugar awareness among older adolescents: Evaluation of a brief nutrition education intervention

\author{
Maria Santalo, University of Victoria
}

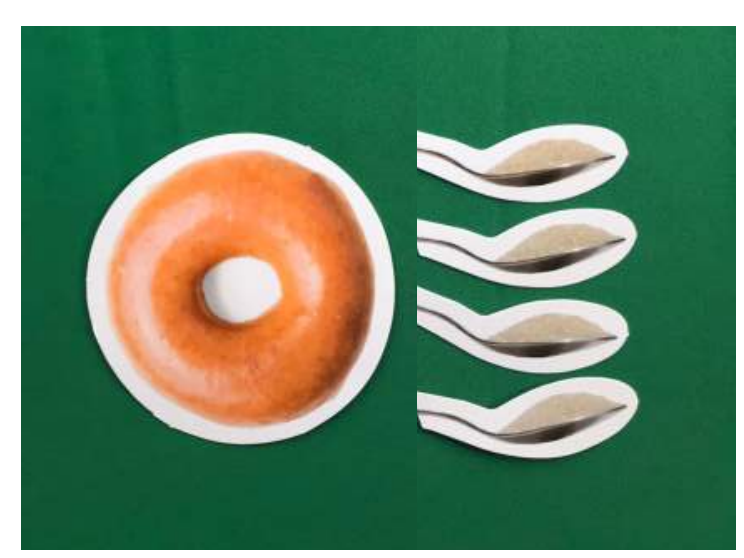

\section{Introduction}

- The overweight and obese population is in continuous growth worldwide with health consequences such as increased risk of developing type 2 diabetes, cardiovascular and liver diseases ${ }^{1}$.

- The World Health Organization recommends to limit the consumption of free sugars, in an attempt to face the problem of overweight and obesity ${ }^{2}$.

- Adolescents. consume the highest amount of added sugar ${ }^{3}$

- More than $60 \%$ of adolescent sugar intake comes from solid food ${ }^{4}$.

- Nutrition literacy has been established as an important step in changing diet behavior ${ }^{5}$.

- Adolescent sugar literacy programs found in literature are focused in Sugar Sweetened Beverages.

- There is a lack in innovative materials for nutrition education with adolescents and the programs are focused mainly in early adolescents ${ }^{6}$.

- Food models have been used mainly to assess the amount of food consumed and for education purposes with children.

\section{Purpose}

The purpose of this research is to examine the impact of a brief nutrition education intervention (focused on sugar content) using two-dimensional food models on the sugar awareness of older adolescents.

\section{Research question}

Does a two 45-minute nutrition session intervention using twodimensional food models significantly increase:

- the knowledge,

- the intention and self-efficacy to consume less added sugar,

- the ability to understand amount of added sugar by reading food labels,

when compared to a non-intervention group of adolescents?

\section{Definitions}

- Free sugar: Mono and disaccharides added to foods and beverages and, sugars naturally present in honey, syrups, fruit juices, and fruit juice concentrates ${ }^{2}$.

- Added sugar: Caloric sweeteners (including honey and undiluted concentrated fruit juice) that are added to foods and beverages as ingredients ${ }^{7}$.

- Sugar awareness: Understanding about the effect of added sugar in health and the knowledge of where and in what amount added sugar is present in food and beverages.

- Sugar literacy: Sugar awareness and skills necessary to assess sugar consumption and change sugar related dietary behaviors.

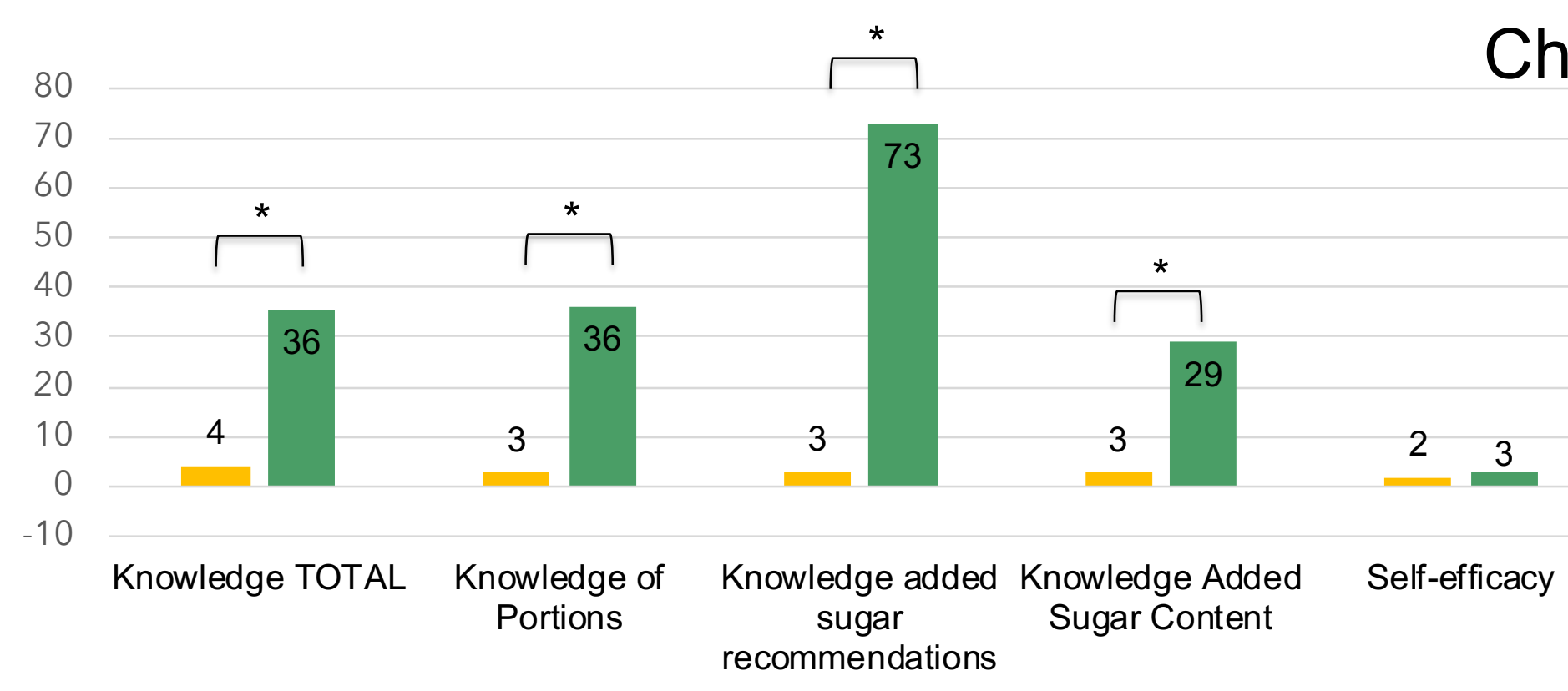

Intervention

203 students grades 9 to 12 from 6 schools in Vancouver lland participated in this randomized comparison trial
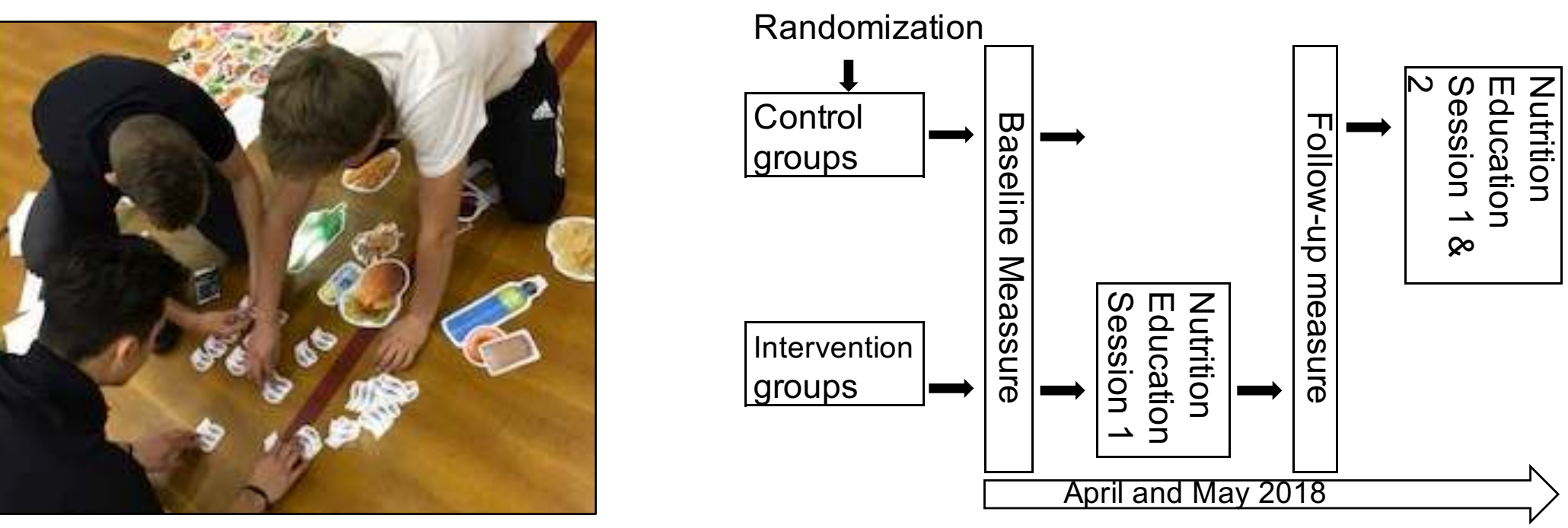

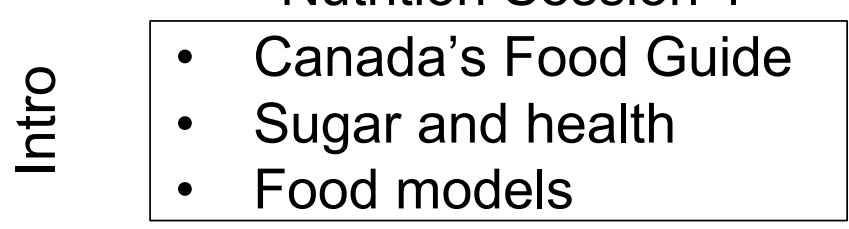

$\bar{\sigma} \sim \boldsymbol{\omega} \cdot$ Represent personal

$\stackrel{\frac{\pi}{2}}{\frac{5}{3}} \frac{5}{0}$ diet

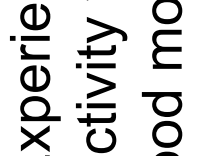

யั

- Design a day Menu according to Canada's Food Guide

- Set goals for personal diet

Data collection and analysis

- 3 demographic questions plus 20 questions from validated questionnaires were used (some questions were adapted).

- Descriptive and repeated measures ANOVA with SPSS 2011 was used to analyze data.

\section{Results}

Low knowledge at baseline. Intervention:

Increased knowledge

Increased ability to interpret sugar content in food

^ Intention to consume less added sugar

$=$ No differences in self-efficacy

A brief sugar nutrition education intervention (focused on sugar content) using two-dimensional food models appears efficacious for increasing sugar literacy among older adolescents.

Change in scores $\bullet$ (baseline to follow up)

${ }^{*}$ Difference was statistically significant

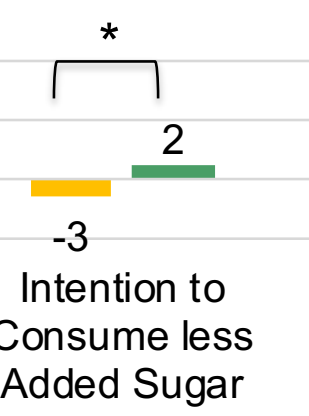

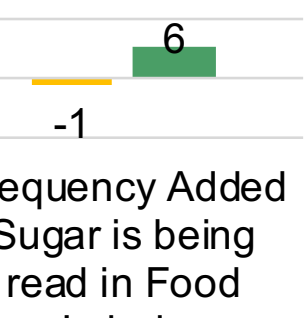

Lead in Food 